\title{
Switch of noninvasive ventilation (NIV) to continuous positive airway pressure (CPAP) in patients with obesity hypoventilation syndrome: a pilot study
}

\author{
Sarah Orfanos ${ }^{1 *}$, Dany Jaffuel ${ }^{2}$, Christophe Perrin ${ }^{3}$, Nicolas Molinari ${ }^{4}$, Pascal Chanez ${ }^{5}$ and Alain Palot ${ }^{5}$
}

\begin{abstract}
Background: Obesity is a major worldwide public health issue. The main respiratory complication stemming from obesity is obesity hypoventilation syndrome (OHS). Most of the OHS patients diagnosed during an exacerbation are treated with non invasive ventilation (NIV). Up to date, no prospective study has demonstrated in real life conditions the feasibility of a systematic protocoled switch of NIV to continuous positive airway pressure (CPAP), once stability is achieved.
\end{abstract}

Methods: In this prospective study, we included stable patients with OHS, with moderate to severe concomitant obstructive sleep apnea (OSA) and without obstructive pulmonary disease, who had been undergoing NIV for more than 2 months. The following measurements were performed, first with NIV and then after the switch to CPAP: diurnal arterial blood gas measurements; nocturnal oximetry and capnometry; mean compliance and AHI; measures of quality of life and quality of sleep.

Results: 22/30 patients accepted to participate in the study and 15/22 patients completed the study. There were no significant differences for pooled data in diurnal alveolar blood gases, nocturnal capnometry $(p=0.534)$, nocturnal oximetry $(p=0.218)$, mean compliance $(p=0.766)$, mean AHI $(p=0.334)$, quality of life or quality of sleep. Eighty percent of the patients treated in this study favored CPAP over NIV.

Conclusion: This pilot study showed in real life conditions the possibility of a systematic switch of NIV to CPAP, in most stable patients with $\mathrm{OHS}$, with similar efficacy on diurnal and nocturnal alveolar gas exchange, quality of life and quality of sleep.

Trial registration: ISRCTN13981084. Registered: 27 February 2017 (retrospectively registered)

Keywords: Obesity hypoventilation syndrome, Non invasive ventilation, Continuous positive airway pressure, Bilevel positive airway pressure, Obstructive sleep apnea

\section{Background}

Obesity is a major worldwide public health issue [1]. The main respiratory complication stemming from obesity is obesity hypoventilation syndrome (OHS). OHS was defined, in 1999, by the American Academy of Sleep Medicine as the association of obesity $\left(\mathrm{BMI} \geq 30 \mathrm{~kg} / \mathrm{m}^{2}\right)$; daytime hypercapnia ( $\mathrm{paCO} 2>45 \mathrm{~mm} \mathrm{Hg}$ ); and sleep disordered

\footnotetext{
* Correspondence: sarah.orfanos@gmail.com

${ }^{1}$ Aix-Marseille University, Faculté de médecine, 27 Boulevard Jean Moulin, 13005 Marseille, France

Full list of author information is available at the end of the article
}

breathing, after excluding other causes of hypoventilation [2]. OHS is a frequent condition with the prevalence of OHS in patients with a $\mathrm{BMI} \geq 35 \mathrm{~kg} / \mathrm{m}^{2}$ being $31 \%$ [3].

$\mathrm{OHS}$ is an underdiagnosed and undertreated condition $[3,4]$, despite the fact that a treatment with positive airway pressure improves quality of life and decreases morbidity in these patients $[3,5]$. The application of a positive airway pressure through noninvasive ventilation (NIV) or continuous positive airway pressure (CPAP), decreases mortality in patients presenting with OHS $[4,6,7]$. 
To date, the main unresolved question is to determine the respective position of NIV and CPAP when treating these patients. If some studies have attempted to determine different OHS's phenotypes to predict the outcome of CPAP therapy [8-10]; only two studies prospectively compared NIV and CPAP treatment in OHS $[5,11]$. To date, the superiority of NIV over CPAP in the treatment of OHS has not been proven. However, 26 to $50 \%$ of patients suffering from OHS are diagnosed in an acute setting [7, 8, 11, 12 ], and most often treated with NIV which remains their usual ventilatory mode for the rest of their life.

Once stability has been reached, the question then arises as to whether NIV should be switched to CPAP. To our knowledge, no prospective trial has evaluated in real life conditions the feasibility of a protocol switching stable patients from NIV to CPAP.

The purpose of this study was to test the feasibility and effect of a standardized protocol, switching patients with OHS, from NIV to CPAP. We hypothesized that there will be no difference in efficacy after switching NIV to CPAP, on AHI, diurnal and nocturnal alveolar gas exchange (daytime arterial blood gas (ABG), nighttime transcutaneous oxygen saturation and transcutaneous measurement of pCO2 (ptCO2)), but also on sleepiness, quality of sleep and quality of life (Epworth Sleepiness Scale (EPS), Pittsburgh Sleep Quality Index (PSQI), Severe Respiratory Insufficiency questionnaire (SRI)).

\section{Methods \\ Patients}

Patients over 18 years old, suffering from OHS, defined as an association of obesity (BMI $\left.\geq 30 \mathrm{~kg} / \mathrm{m}^{2}\right)$ and daytime hypercapnia ( $\mathrm{paCO} 2>45 \mathrm{~mm} \mathrm{Hg}$ ), who had been undergoing NIV for more than 2 months, were prospectively recruited from February 2015 to February 2016 in the pulmonology wards of the university hospital (hôpital Nord) and the military hospital (hôpital Laveran) in Marseille, France. To be included, patients had to be clinically stable for at least 4 weeks before enrollment in the study (no hospitalization or emergency admission). The exclusion criteria were: patients who presented hypercapnia secondary to other causes (obstructive pulmonary diseases $(\mathrm{FEV} 1 / \mathrm{FVC}<70)$, interstitial lung diseases, neuromuscular or chest wall diseases, severe hypothyroidism or congenital central hypoventilation syndrome), and patients unable to give informed consent.

This trial respected the ethical principles of the declaration of Helsinki and was approved by the local ethic committee of the Assistance Publique Hôpitaux de Marseille (AP-HM). Written informed consent was obtained from all patients. The use of data was approved by the Data Privacy Officer of the hospitals (AP-HM).

\section{Study protocol}

Patients who had been undergoing NIV for at least the last 2 month, were prospectively included in the study by the investigators, during a follow-up outpatient clinic.

During this clinic, the following clinical information were collected: history of the diagnosis of OHS, occurrence of acute exacerbations, NIV settings: brand, mode, respiratory rate (RR), expiratory positive airway pressure (EPAP), inspiratory positive airway pressure (IPAP) and interfaces.

The settings were in accordance with the American Academy of Sleep Medicine (AASM) recommendations [13]. NIV settings were optimized in order to avoid patient-ventilator dyssynchrony and to correct remaining respiratory events during sleep, according to the recommendations of the somnoNIV group [14].

Quality of life was evaluated using the SRI questionnaire; daytime sleepiness was evaluated using the ESS questionnaire; and quality of sleep was evaluated using the PSQI questionnaire.

Finally, an ABG analysis was performed, during the consultation, between 10 and 12 am in an awake, seated patient, at rest for at least $10 \mathrm{~min}$.

During one night with NIV, at home, the patient underwent: (i) nocturnal oximetry using a pulse oxim-

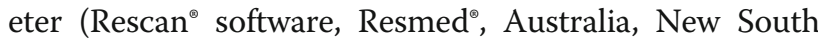
Wales, Bella-Vista), on which were calculated the percentage of recording time with $\mathrm{SaO} 2<90 \%$ ( $\mathrm{RT}<90 \%$ ) and the oxygen desaturation index (ODI) (defined as the number of time per hour the oxygen saturation drops by $\geq 4 \%$ ); (ii) nocturnal transcutaneous capnometry using a transcutaneous capnometer (Tosca $500^{\circ}$, Radiometer ${ }^{\odot}$, Crawley, UK), on which was calculated the mean ptCO2; (iii) a report of the mean compliance, the median leak, the mean tidal volume and the mean AHI index of the last month spent with NIV was extracted (Rescan ${ }^{\circ}$ software for Resmed ${ }^{\oplus}$, Australia New South Wales, BellaVista NIV and Directview ${ }^{\oplus}$ for Philips-Respironics ${ }^{\oplus}$, Murrysville, Pennsylvania, USA NIV).

After undergoing these procedures, the patients underwent NIV withdrawal for seven nights. At the end of this week, pulmonary function tests (PFT) were performed according to the American Thoracic Society and European Respiratory Society 2005 guidelines [15], and an overnight polygraphy during spontaneous breathing was performed (Alice PDx ${ }^{\oplus}$, Philips-Respironics ${ }^{\oplus}$, Murrysville, Pennsylvania). Polygraphic recordings were analyzed by a blinded pulmonologist certified in sleep medicine, respiratory events were scored manually according to the AASM 2012 manual [16]. The AHI, RT $<90 \%$ and ODI on this polygraphy during spontaneous breathing were reported. At this stage of the trial, patients exhibiting obstructive ventilatory disorder (FEV1/FVC $<70)$ or mild OSA (AHI $<15 /$ h), were not switched to CPAP and continued their 
treatment with NIV. All the other patients were switched to CPAP, the settings were as follow: constant pressure, EPAP $2 \mathrm{~cm} \mathrm{H}_{2} \mathrm{O}$ above the EPAP of the previous NIV to compensate for the absence of an IPAP on the CPAP, settings were not adjusted until measurements were repeated more than a month after this switch.

After more than a month with CPAP; SRI, ESS and PSQI questionnaires were reevaluated. An ABG analysis was performed in the same conditions as previously described. During one night with CPAP, at home, the patient underwent: (i) nocturnal oximetry; (ii) nocturnal transcutaneous capnometry; (iii) a report of the mean compliance, the median leak and the mean $\mathrm{AHI}$ index of the last month spent with CPAP was extracted. (Fig. 1).

As a secondary analysis, we analyzed the characteristics of patients failing NIV and CPAP using Salord et al. criteria to define failure: more than $30 \%$ of recording time spent below $90 \%$ saturation or paCO2 $>45 \mathrm{~mm} \mathrm{Hg}$,

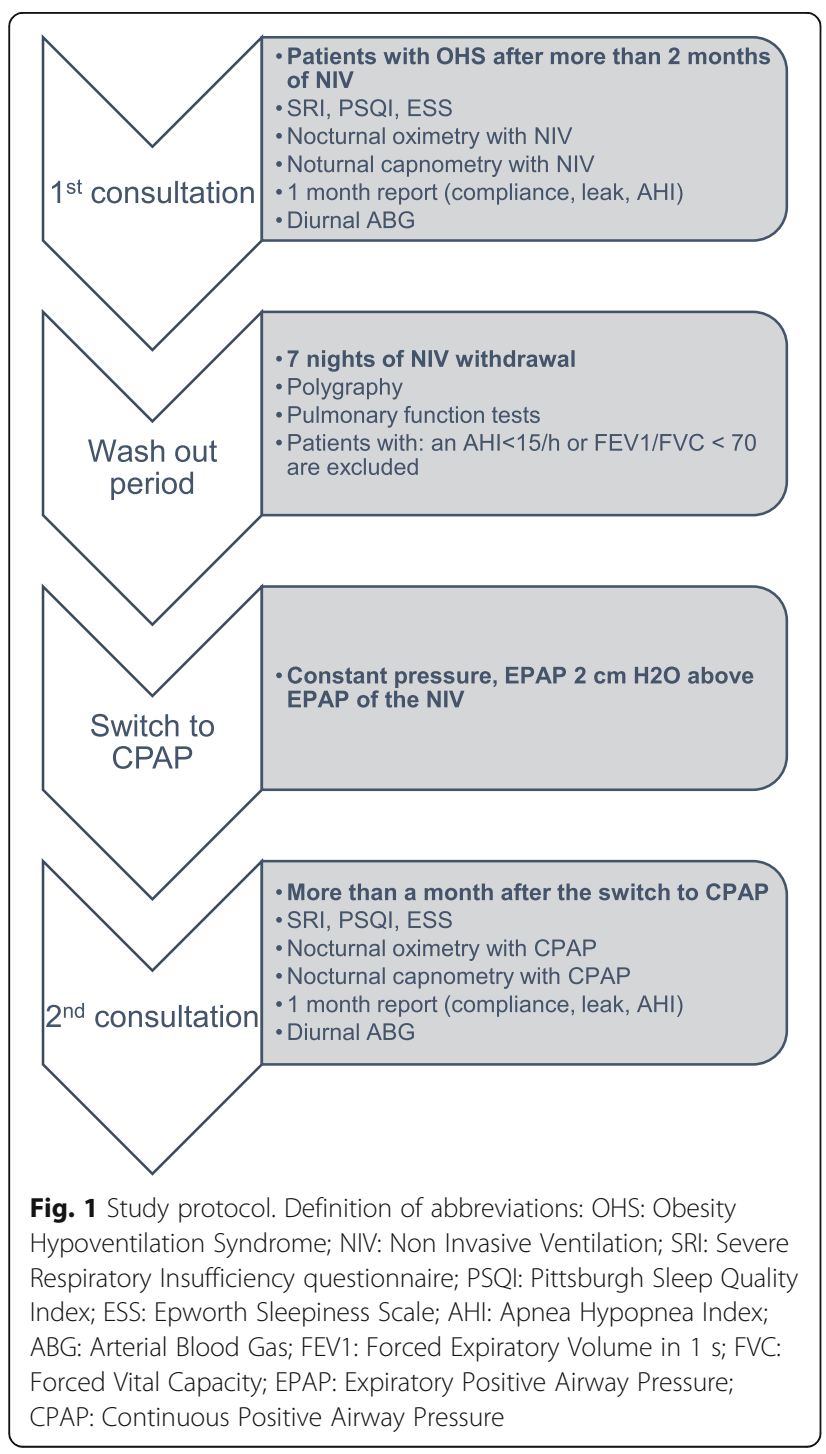

to determine if a characteristic could be used as a prognostic factor of ventilation failure [10].

\section{Statistical analysis}

Continuous data were expressed in means and SDs and categorical data were expressed as percentages and absolute.

Normality was assessed using Shapiro-Wilk normality test. Intra-group changes in variables measured at first with NIV and after the switch to CPAP were assessed using paired t-tests or non-parametric Wilcoxon tests depending on normality.

When comparing two independent groups (NIV failure, NIV success), unpaired t-tests or non-parametric MannWhitney tests depending on normality were used for continuous variables and $\mathrm{x}^{2}$ tests for categorical variables.

\section{Results}

\section{Study population}

Twenty-two patients were included. Five dropped-out due to unwillingness to be hospitalized for the polygraphy prior to the switch to CPAP. Two patients were excluded prior to the switch, one had an obstructive syndrome on the PFT, and the other had an AHI $<15 / \mathrm{h}$ on the polygraphy. Fifteen of the 30 patients who were initially selected, were switched to CPAP (Fig. 2).

Anthropometric characteristics of the patients are shown in Table 1.

Seven nights after the NIV was discontinued, polygraphy of the weaning period showed a high mean AHI $(\mathrm{AHI}=53.5 / \mathrm{h} \pm 26.9)$ and the mean $\mathrm{RT}<90 \%$ was $21.8 \%$ (24.2) (Table 2).

Regarding settings, all NIV were in spontaneous timed (ST) mode, mean EPAP was $7.7 \pm 1.8 \mathrm{~cm} \mathrm{H2O}$, mean IPAP was $18.3 \pm 3 \mathrm{~cm} \mathrm{H} 2 \mathrm{O}$, mean back-up RR was 13.9 $\pm 1.8 \mathrm{cycles} / \mathrm{min}$. Two patients received additional oxygen therapy, flow rates were not modified after switching to CPAP. (Table 3).

\section{Effect of the switch to CPAP on the measured data}

There were no significant differences, after the switch to CPAP, in mean AHI, diurnal and nocturnal alveolar gas exchanges (diurnal ABG, nocturnal oximetry and transcutaneous capnometry), median leaks and compliance (Table 4, Fig. 3).

Regarding ESS, SRI and PSQI scores, the only significant difference was in the ESS, which was lower after the switch to CPAP $(8.21 \pm 5.54$ vs $4.29 \pm 3.43)(p=$ $0.004)$. There were no significant differences in the SRI and all its subscales and in the PSQI and all its components between before and after the switch to CPAP. (Table 5, Fig. 4).

At the end of the trial, through a self-administered questionnaire, the patients' preference for one mode of ventilation was determined. Twelve patients (80\%) 


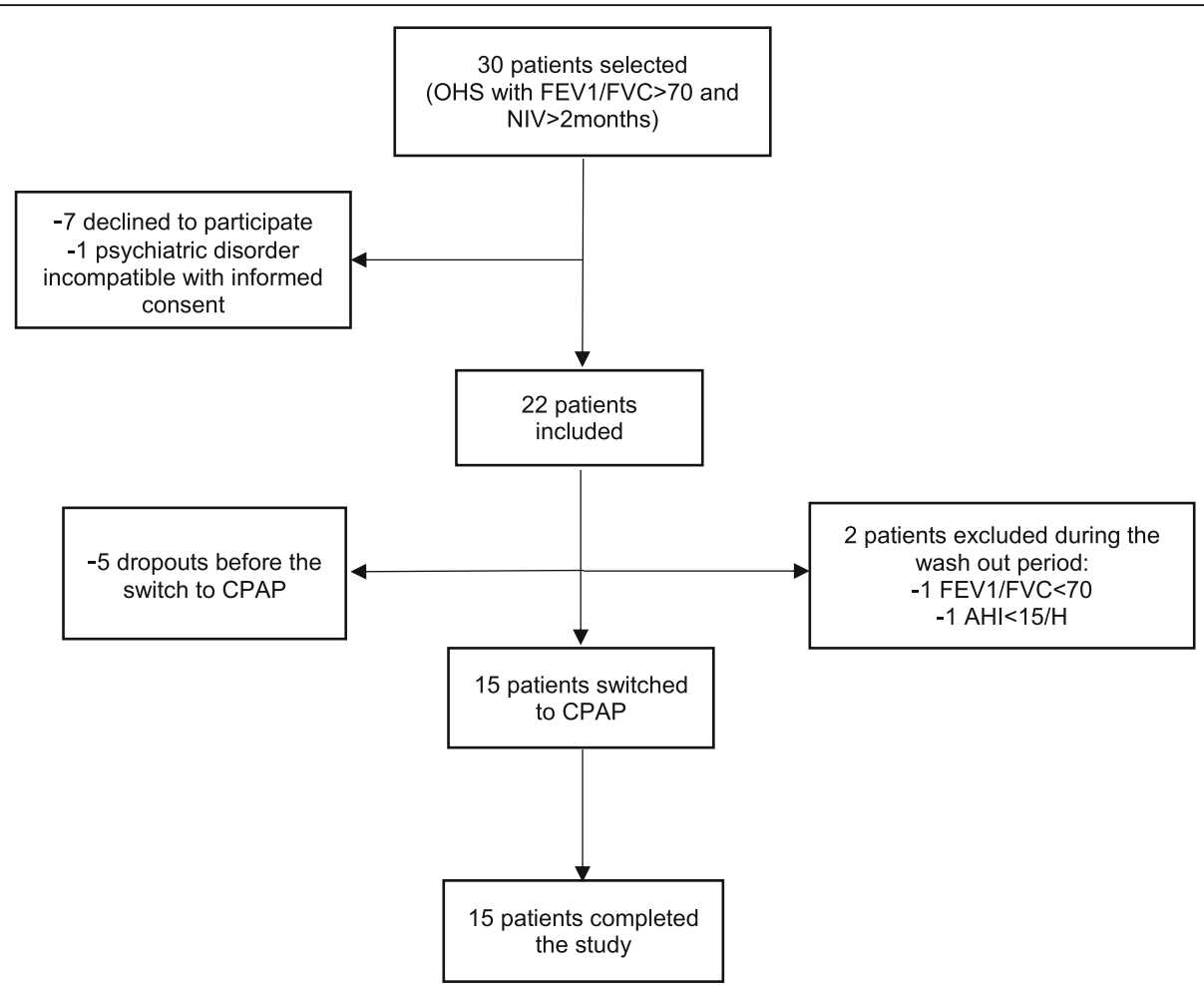

Fig. 2 Flow chart. Definition of abbreviations: OHS: Obesity Hypoventilation Syndrome; FEV1: Forced Expiratory Volume in 1 s; FVC: Forced Vital Capacity; NIV: Non Invasive Ventilation; CPAP: Continuous Positive Airway Pressure; AHI: Apnea Hypopnea Index

reported that they preferred CPAP over NIV and wanted to continue their treatment with CPAP. Two patients (13\%) had no preference. Only one patient wanted to discontinue CPAP and go back to NIV, despite the fact that there were no objective criteria of deterioration during CPAP treatment.

\section{Safety}

None of the patients presented with clinical deterioration after more than a month of CPAP and needed to be switched back to NIV. Two patients were admitted in the intensive care unit during the trial, due to causes unrelated to the mode of ventilation. One presented with acute renal failure complicated by symptomatic hyperkalemia and the other with left heart failure secondary to non-compliance to diuretics.

\section{Cost}

When taking into account French social security reimbursement rate, NIV cost was $63.16 €$ per week and CPAP cost was $18 €$ per week. This switch may allow an economy of $2348 €$ per year per patient.

\section{NIV failure}

When analyzing the characteristics of the patients failing NIV, according to Salord's criteria: more than $30 \%$ of recording time spent below $90 \%$ saturation or paCO2 > $45 \mathrm{~mm} \mathrm{Hg}$; BMI was the only factor associated with NIV failure $(p=0.014)$ (Table 6).

\section{Discussion}

This prospective open trial, testing the feasibility of a protocol switching NIV to CPAP in stable patients in real life conditions, suggests the possibility of this switch in patients suffering from OHS with an $\mathrm{AHI} \geq 15 / \mathrm{h}$ and without pulmonary obstructive disease. In these conditions, we reported no significant treatment effect differences between before and after the switch to CPAP in diurnal and nocturnal alveolar gas exchange, mean AHI, mean compliance, quality of sleep and quality of life. In addition, $80 \%$ of patients preferred CPAP over NIV.

The pathophysiology of OHS is complex and multifactorial. Eventually leading to chronic, diurnal hypercapnia resulting from an imbalance between apnea and interapnea periods and deficiency of the compensatory mechanisms to unload the carbon dioxide in excess $[17,18]$. This suggests the need to determine different phenotypes of OHS, and therefore adapt therapeutic intervention according to the phenotype. Therefore we have chosen in this trial, to exclude from the study, patients with an $\mathrm{AHI}<15 / \mathrm{h}$, speculating that CPAP mechanism to treat hypoventilation was mostly due to the correction of apneas and hypopneas. Indeed, if CPAP is not 
Table 1 Baseline characteristics

\begin{tabular}{ll}
\hline Baseline characteristics & $n=15$ \\
\hline Sex (male) & $5(33.3 \%)$ \\
Age (years) & $68.8 \pm 11.2$ \\
BMI (kg/m $\left.{ }^{2}\right)$ & $41.7 \pm 7.16$ \\
Neck circumference $(\mathrm{cm})$ & $43.6 \pm 4.5$ \\
Waist/Hip ratio & $0.95 \pm 0.08$ \\
pH on ABG before initiating NIV & $7.38 \pm 0.06$ \\
paCO2 on ABG before initiating NIV (mmHg) & $57.7 \pm 7.6$ \\
paO2 on ABG before initiating NIV (mmHg) & $62.6 \pm 18.8$ \\
HCO3- before initiating NIV (mmol/L) & $31.8 \pm 5.1$ \\
Actual or past smokers & $7(46.7 \%)$ \\
Pack per year & $38.1 \pm 30.9$ \\
Cardiovascular comorbidities & $13(86.7 \%)$ \\
Hypertension & $12(80 \%)$ \\
Dyslipidemia & $12(80 \%)$ \\
Diabetes & $9(60 \%)$ \\
Cardiac disease & $12(80 \%)$ \\
Ischemic cardiac disease & $5(33.3 \%)$ \\
Cardiac arrhythmia & $5(33.3 \%)$ \\
LVEF (\%) & $56 \pm 14.3$ \\
Diastolic dysfunction & $8(61.5 \%)$ \\
Diagnosis made during an exacerbation & $12(80 \%)$ \\
\hline Data are expressed in $n$
\end{tabular}

Data are expressed in $\mathrm{n}(\%)$ and mean \pm standard deviation

Definition of abbreviations: $B M I$ body mass index, $A B G$ arterial blood gas, NIV non invasive ventilation, $L V E F$ left ventricular ejection fraction

intrinsically the most efficient treatment of hypoventilation, it is still effective on most of the pathophysiological mechanisms leading to OHS by decreasing upper airways resistance, increasing central response to hypercapnia and hypoxemia [19, 20], increasing lung volumes, treating atelectasis, decreasing intrinsic PEEP [21]. This is the reason why CPAP is efficient in most patients with OHS associated with OSA.

Table 2 Wash out period: pulmonary function tests and polygraphy

\begin{tabular}{ll}
\hline PFT and Polygraphy (during withdrawal) & $n=15$ \\
\hline FEV1/FVC (\%) & $79.5 \pm 7.4$ \\
FEV1 (\%) & $69.2 \pm 16.4$ \\
FVC (\%) & $70.2 \pm 17.3$ \\
AHI (n/h) & $53.5 \pm 26.9$ \\
RT $<90 \%(\%)$ & $21.8 \pm 24.2$ \\
ODI $\geq 4 \%(n / h)$ & $42.1 \pm 39.5$
\end{tabular}

Data are expressed in mean \pm standard deviation Definition of abbreviations: PFT pulmonary function test, FEV1 forced expiratory volume in $1 \mathrm{~s}$, FVC forced vital capacity, AHI apnea hypopnea index, $R T<90 \%$ percentage of recording time below $90 \%$ saturation, ODI oxygen desaturation index
Table 3 NIV and CPAP settings

\begin{tabular}{|c|c|}
\hline NIV and CPAP settings & $n=15$ \\
\hline NIV mode & ST \\
\hline NIV EPAP (cm H2O) & $7.7 \pm 1.8$ \\
\hline NIV IPAP (cm H2O) & $18.3 \pm 3$ \\
\hline NIV RR (n/min) & $13.9 \pm 1.8$ \\
\hline NIV VT (ml) & $508.3 \pm 264$ \\
\hline CPAP EPAP $(\mathrm{cm} \mathrm{H2O})$ & $9.8 \pm 1.4$ \\
\hline Oronasal mask & $10(66.7 \%)$ \\
\hline Nasal mask & $3(20 \%)$ \\
\hline Nasal pillows & $2(13.3 \%)$ \\
\hline
\end{tabular}

Two prospective randomized trials have compared the efficacy of CPAP and NIV in patients with OHS $[5,11]$. These two prospective studies corroborate the fact that patients with OHS and concomitant OSA can be treated with CPAP. Piper and colleagues did not find a significant difference, at three months, in daytime paCO2 between the two groups NIV and CPAP [11]. However in this study, nine of the 45 patients initially selected were excluded from the trial, due to persisting nocturnal hypoxemia or nocturnal hypercapnia on the first night of CPAP titration. Treating hypoventilation in some phenotypes may take up to a few weeks [22], excluding patients after one night of CPAP titration is debatable. Masa and colleagues confirmed the absence of a significant difference between NIV and CPAP, at two months, in diurnal paCO2 [5]. However, only NIV and not CPAP significantly improved paCO2 compared to the control group. An improvement was only noted for patients

Table 4 Measured data with NIV and one month after the switch to CPAP

\begin{tabular}{lccc}
\hline Measured data & NIV & CPAP & $P$ value \\
\hline $\mathrm{Pa} \mathrm{CO} 2(\mathrm{~mm} \mathrm{Hg})$ & $40.4 \pm 5.1$ & $40.9 \pm 6.7$ & 0.323 \\
$\mathrm{~Pa} \mathrm{O2} \mathrm{(mm} \mathrm{Hg)}$ & $73.3 \pm 8.8$ & $74.4 \pm 8.8$ & 0.235 \\
$\mathrm{pH}$ & $7.43 \pm 0.04$ & $7.43 \pm 0.03$ & 0.508 \\
$\mathrm{HCO} 3 \quad(\mathrm{mmol} / \mathrm{L})$ & $26.3 \pm 3.14$ & $26.6 \pm 3.6$ & 0.511 \\
$\mathrm{RT}<90 \%(\%)$ & $28.1 \pm 27.2$ & $20.5 \pm 25.2$ & 0.218 \\
ODI $\geq 4 \%$ (n/h) & $11.92 \pm 17.31$ & $10 \pm 9.41$ & 0.285 \\
Transcutaneous pCO2 (mm Hg) & $49.9 \pm 10.12$ & $48.4 \pm 5.14$ & 0.534 \\
AHI (n/h) & $5.99 \pm 10.96$ & $5.49 \pm 7.84$ & 0.334 \\
Leaks (L/sec) & $3.8 \pm 10.09$ & $2.9 \pm 6.1$ & 0.844 \\
Compliance (h) & $6.73 \pm 2.66$ & $6.8 \pm 2.27$ & 0.766 \\
\hline
\end{tabular}

Data are expressed in mean (standard deviation)

Definition of abbreviation: NIV non invasive ventilation, CPAP continuous positive airway pressure, $R T<90 \%$ percentage of recording time below $90 \%$ saturation, $O D I$ oxygen desaturation index, $A H I$ apnea hypopnea index 

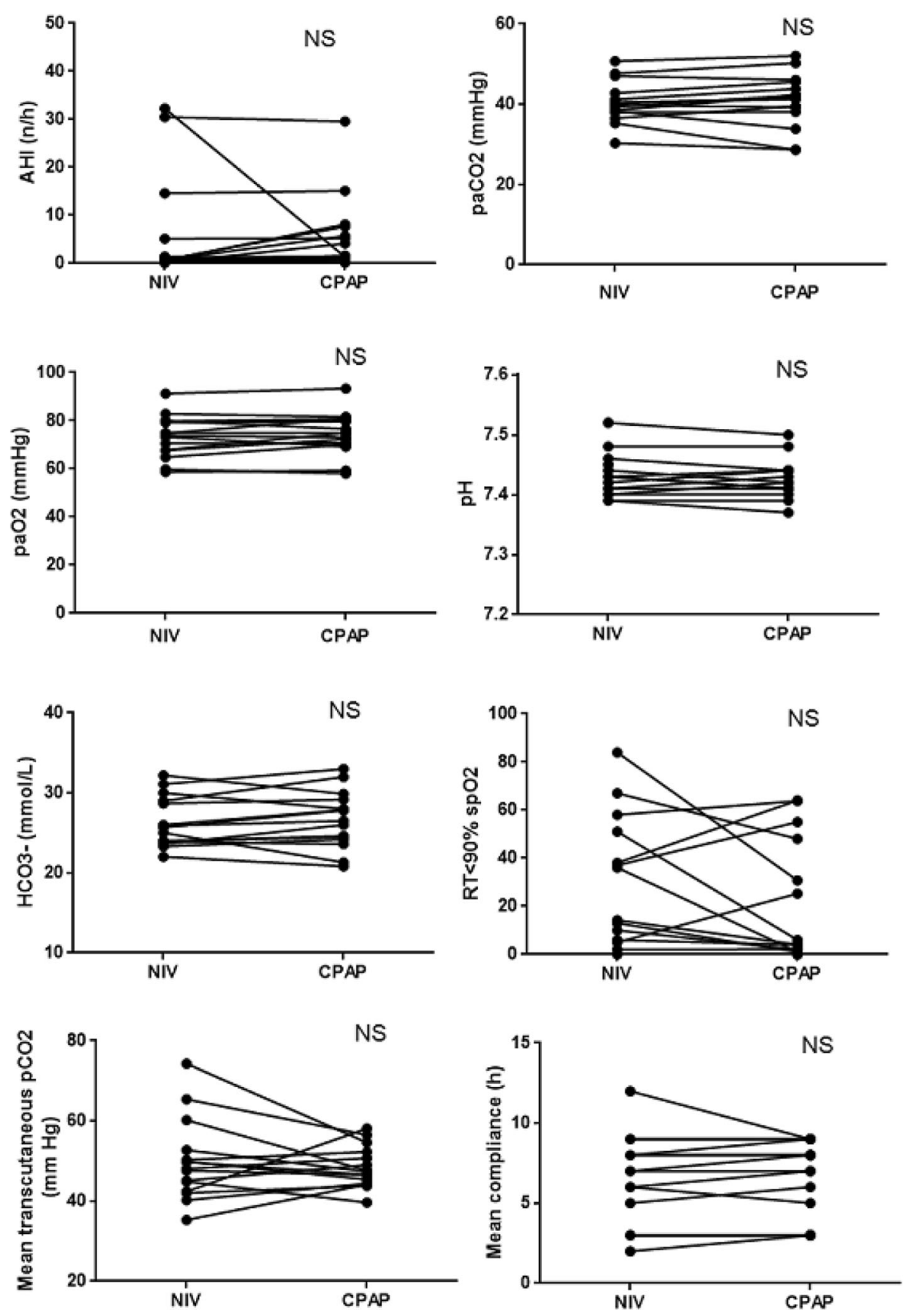

Fig. 3 Measurement of mean AHI; diurnal and nocturnal alveolar gas exchange parameters and mean compliance, in 15 patients, with NIV and one month after the switch to CPAP. Definition of abbreviations: NIV: Non Invasive Ventilation; CPAP: continuous positive airway pressure; AHI: Apnea Hypopnea Index; RT < 90\% spO2: percentage of recording time below 90\% saturation; NS: no significant difference $p>0.05$

using CPAP more than $4 \mathrm{~h}$ per night. Moreover, the authors found a significant difference, favoring NIV, on the 6 min' walk test and respiratory function tests.

The heterogeneity in responses to CPAP, led some studies to try to define phenotypes of OHS and predictor factors of CPAP failure. Therefore, patients exhibiting a higher BMI, a higher percentage of recording time below 90\% saturation, a lower AHI, or a decreased forced vital capacity could be at higher risk of CPAP failure, even though these predictor factors are not constantly found in all studies [8-10]. In our study, we found that only BMI had a significant association with CPAP or NIV failure (Table 6). When using the two criteria defining failure in Salord and colleagues study [10] (more than $30 \%$ of recording time spent below $90 \%$ saturation or paCO2 $>45 \mathrm{~mm} \mathrm{Hg}$ ), in our study $8 / 15$ patients would 
Table 5 ESS, PSQI and its components, SRI and its subscales scores with NIV and one month after the switch to CPAP

\begin{tabular}{lrrl}
\hline Scores & \multicolumn{1}{l}{ NIV } & \multicolumn{1}{l}{ CPAP } & $P$ value \\
\hline ESS & $8.21 \pm 5.54$ & $4.29 \pm 3.43$ & $0.004^{*}$ \\
PSQI & $6.86 \pm 4.77$ & $6.14 \pm 3.65$ & 0.311 \\
Componant 1 & $1.07 \pm 0.92$ & $0.86 \pm 0.53$ & 0.500 \\
Componant 2 & $0.79 \pm 1.37$ & $1.29 \pm 1.27$ & 0.250 \\
Componant 3 & $0.93 \pm 0.83$ & $0.93 \pm 1.07$ & 1 \\
Componant 4 & $0.5 \pm 0.85$ & $0.57 \pm 0.85$ & 0.531 \\
Componant 5 & $1.29 \pm 0.61$ & $1.21 \pm 0.43$ & 1 \\
Componant 6 & $1.21 \pm 1.48$ & $0.43 \pm 1.09$ & 0.125 \\
Componant 7 & $1 \pm 1.18$ & $0.86 \pm 1.03$ & 0.766 \\
SRI & $62.4 \pm 19.46$ & $66.5 \pm 17.32$ & 0.153 \\
SRI RC & $70 \pm 21.61$ & $71.87 \pm 21.58$ & 0.529 \\
SRI PF & $48.61 \pm 28.33$ & $46.13 \pm 29.17$ & 0.404 \\
SRI AS & $54.83 \pm 28.80$ & $64.54 \pm 22.04$ & 0.135 \\
SRI SF & $70.83 \pm 21.30$ & $75 \pm 19.81$ & 0.682 \\
SRI ANX & $69.67 \pm 16.74$ & $70.14 \pm 18.02$ & 0.968 \\
SRI WB & $61.31 \pm 28.63$ & $73.21 \pm 21.89$ & 0.240 \\
SRI SR & $61.54 \pm 23.52$ & $64.38 \pm 22.25$ & 0.675 \\
\hline Da are & & &
\end{tabular}

Data are expressed in mean (standard deviation)

Definition of abbreviations: NIV non invasive ventilation, CPAP continuous positive airway pressure, ESS epworth sleepiness scale, PSQ/ Pittsburgh sleep quality index, $S R I$ severe respiratory insufficiency questionnaire, $R C$ respiratory complaints, $P F$ physical functioning, $A S$ attendant symptoms, $S F$ social functioning, $A N X$ anxieties, $W B$ well being, $S R$ social relationships

*means significance with $p$ value $<0.05$ be categorized as NIV failure and 7/15 as CPAP failure. Interestingly, the seven patients failing CPAP were the same patients who were failing NIV before the switch. We do not think this failure can be attributed to NIV or CPAP settings in our study. Mean EPAP was $8 \mathrm{~cm} \mathrm{H} 2 \mathrm{O}$, mean IPAP was $18 \mathrm{~cm} \mathrm{H2O,} \mathrm{back-up} \mathrm{RR} \mathrm{was} 14$ cycles/ min, all NIV were in ST mode. Concerning CPAP, mean EPAP was $9.8 \mathrm{~cm} \mathrm{H} 2 \mathrm{O}$. These pressures are concordant with previous trials $[5,11]$.

Our study has some limitations. First of all it can be argued that the sample size in our study (15 patients) could be insufficient to detect a significant difference after the switch to CPAP, and that this absence of difference could be due to a lack of power. This study does not have the pretention to assert the absence of difference between NIV and CPAP at a larger scale, but describes a feasible protocol for most sleep clinic, to switch stable patients with OHS from NIV to CPAP. As suggested by our preliminary results, for some patients, the switch of NIV to CPAP can be deleterious emphasizing the need for a careful monitoring. A second limitation is that the results of our study can only be extended to patients with OHS and concomitant OSA with AHI $\geq 15 / \mathrm{h}$, without obstructive pulmonary diseases, and cannot be used when treating patients in an acute state or with mild OSA. These drastic selection criteria aimed at targeting CPAP responders' phenotypes and the limited number of patients included in the study could partly explain the stable diurnal capnia after the switch to CPAP in all of our patients. The
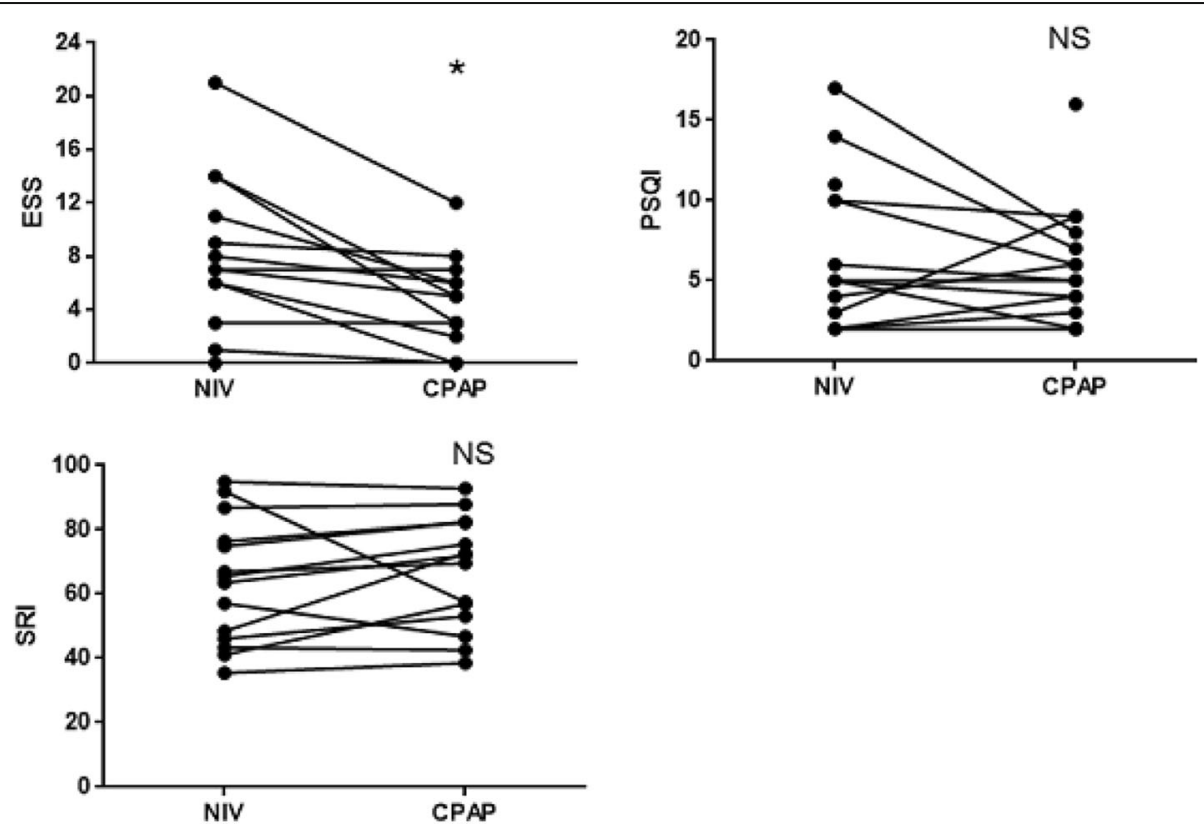

Fig. 4 Changes in ESS, PSQI and SRI scores one month after the switch to CPAP. Definition of abbreviations: NIV: Non Invasive Ventilation; CPAP: Continuous Positive Airway Pressure; ESS: Epworth Sleepiness Scale, PSQI: Pittsburgh Sleep Quality Index; SRI: Severe Respiratory Insufficiency questionnaire; NS: no significant difference; *: significant difference $p<0.05$ 
Table 6 NIV failure predictor factors

\begin{tabular}{llll}
\hline Clinical characteristics & NIV failure $(n=8)$ & NIV success $(n=7)$ & $P$ values \\
\hline Age (years) & $67.12 \pm 8.64$ & $70.71 \pm 14.04$ & 0.555 \\
BMI $\left(\mathrm{kg} / \mathrm{m}^{2}\right)$ & $46.54 \pm 4.84$ & $36.2 \pm 5.07$ & $0.014^{*}$ \\
Neck circumference $(\mathrm{cm})$ & $45.12 \pm 5.64$ & $41.93 \pm 1.97$ & 0.179 \\
Waist/Hip ratio & $0.94 \pm 0.08$ & $0.97 \pm 0.09$ & 0.448 \\
paCO2 at diagnosis (mm Hg) & $57.12 \pm 8.56$ & $58.29 \pm 6.97$ & 0.780 \\
Cardiovascular comorbidities & $7(87.5 \%)$ & $6(85.7 \%)$ & 1 \\
FVC (\%) & $68.07 \pm 19.46$ & $70.49 \pm 13.48$ & 0.788 \\
AHI (n/h) & $63.09 \pm 28.53$ & 42.5421 .76 & 0.145 \\
RT $<90 \%(\%)$ & $26.45 \pm 28.55$ & $16.54 \pm 18.89$ & 0.450 \\
Diagnosis during an exacerbation & $6(75 \%)$ & $6(85.7 \%)$ & 0.605
\end{tabular}

Data are expressed in $\mathrm{n}(\%)$; mean (standard deviation)

Definition of abbreviations: NIV non invasive ventilation, BMI body mass index, FVC forced vital capacity, $A H I$ apnea hypopnea index, $R T<90 \% \%$ of recording time below $90 \%$ saturation

treatment of patients with OHS with pure hypoventilation and without OSA, is seldom discussed in scientific literature, and these patients still benefit from NIV in most teams [23]. For ethical reasons, we decided not to switch to CPAP, patients with mild OSA (AHI $<15 / \mathrm{h}$ ), to not deprive these patients with previous acute exacerbation from an effective therapy.

To eliminate NIV persistent effect, we introduced in the protocol, a withdrawal from NIV for seven nights before starting CPAP, and reevaluated patients more than a month after starting CPAP. It is possible that not all persistent effects attributable to NIV were eliminated by this withdrawal [24]. An ideal protocol would have been to randomize patients into three groups: one group pursuing NIV, one group switching to CPAP and one group stopping ventilation; but this protocol did not seem ethical for patients withdrawn from ventilation, considering that a higher mortality rate has been reported in untreated patients with OHS [4, 6, 7]. In our study, 12 out of 15 patients were diagnosed with OHS and started on NIV, following an episode of exacerbation. This raises the question as to whether the diagnosis of OHS was made in excess in patients with normocapnic OSA undergoing an exacerbation. This assertion is however contradicted by the fact that nine out of the 12 patients diagnosed with $\mathrm{OHS}$ following an exacerbation, had high bicarbonates level (HCO3->28 mmol/l), in favor of chronic hypercapnia [25]. When measuring the AHI, we used the built-in software available in the NIV and the CPAP. Although the accuracy of these systems is debatable, recent studies are in favor of a good reliability of these built-in softwares [26].

\section{Conclusion}

In conclusion, this preliminary study showed in real life conditions, the possibility of a systematic switch of NIV to CPAP in most stable patients with OHS and a concomitant OSA, with similar efficacy on diurnal and nocturnal alveolar gas exchange, quality of life and quality of sleep. These results need to be confirmed by a larger trial that could also evaluate the clinical consequences of this switch in the longer term, especially cardio-vascular consequences.

\section{Abbreviations}

AASM: American Academy of Sleep Medicine; ABG: Arterial blood gas; AHI: Apnea hypopnea index; BMI: Body mass index; CPAP: Continuous positive airway pressure; EPAP: Expiratory positive airway pressure; EPS: Epworth sleepiness scale; FEV1: Forced expiratory volume in 1 second; FVC: Forced vital capacity; HCO3-: Bicarbonates; IPAP: Inspiratory positive airway pressure; NIV: Noninvasive ventilation; ODI: Oxygen desaturation index; OHS: Obesity hypoventilation syndrome; OSA: Obstructive sleep apnea; PaCO2: Partial pressure of arterial carbon dioxide; PaO2: Partial pressure of arterial oxygen; PFT: Pulmonary function tests; PSQI: Pittsburgh sleep quality index; PtCO2: Transcutaneous measurement of carbon dioxide; RR: Respiratory rate; RT < 90\%: percentage of recording time with $\mathrm{SaO} 2<$ 90\%; SaO2: Oxygen saturation; SD: Standard deviation; SRI: Severe respiratory insufficiency questionnaire; ST: Spontaneous timed mode

\section{Acknowledgements \\ The authors would like to thanks ARARD for its help in the study.}

\section{Funding}

No funding.

\section{Availability of data and materials}

All results from oximetry, capnometry, polygraphy, pulmonary function tests, arterial blood gases: are available at the university hospital of Marseille (APHM).

\section{Authors' contributions}

SO designed study, collected data, analyzed data, wrote manuscript. DJ, CP and PC designed study and revised the manuscript. NM analyzed data and did statistical analysis. AP designed study, collected data, analyzed data and revised the manuscript. All authors read and approved the final manuscript.

\section{Competing interests}

The authors declare that they have no competing interests.

Consent for publication

Not applicable. 


\section{Ethics approval and consent to participate}

This trial respected the ethical principles of the declaration of Helsinki and was approved by the local ethic committee of the Assistance Publique Hôpitaux de Marseille (AP-HM). Written informed consent was obtained from all patients. The use of data was approved by the Data Privacy Officer of the hospitals (AP-HM).

\section{Publisher's note}

Springer Nature remains neutral with regard to jurisdictional claims in published maps and institutional affiliations.

\section{Author details}

${ }^{1}$ Aix-Marseille University, Faculté de médecine, 27 Boulevard Jean Moulin, 13005 Marseille, France. ${ }^{2}$ Département de Pneumologie, Hôpital Arnaud de Villeneuve, CHRU de Montpellier, 371 Avenue du Doyen Giraud, 34295 Montpellier, Cedex 5, France. ${ }^{3}$ Service de Pneumologie, Pôle des Spécialités Médicales, Centre Hospitalier de Cannes, 15 avenue des Broussailles, 06401 Cannes, France. ${ }^{4}$ INSERM U1046, Université de Montpellier 1 et Département Médical d'Information, Centre Hospitalier Universitaire, 34090 Montpellier, France. ${ }^{5}$ Clinique des Bronches, Allergies et du Sommeil, Assistance Publique Hôpitaux de Marseille, France et INSERM U1067, CNRS UMR 7333 Aix Marseille Université, 13015 Marseille, France.

\section{Received: 23 September 2016 Accepted: 4 March 2017} Published online: 14 March 2017

\section{References}

1. Ng M, Fleming T, Robinson M, Thomson B, Graetz N, Margono C, et al. Global, regional, and national prevalence of overweight and obesity in children and adults during 1980-2013: a systematic analysis for the Global Burden of Disease Study 2013. Lancet. 2014;384(9945):766-81.

2. Sleep-related breathing disorders in adults: recommendations for syndrome definition and measurement techniques in clinical research. The Report of an American Academy of Sleep Medicine Task Force. Sleep. 1999;22(5):667-89.

3. Nowbar S, Burkart KM, Gonzales R, Fedorowicz A, Gozansky WS, Gaudio JC, et al. Obesity-Associated hypoventilation in hospitalized patients: prevalence, effects, and outcome. Am J Med. 2004;116(1):1-7.

4. Pépin J-L, Borel J-C, Janssens J-P. Obesity hypoventilation syndrome: an underdiagnosed and undertreated condition. Am J Respir Crit Care Med. 2012;186(12):1205-7.

5. Masa JF, Corral J, Alonso ML, Ordax E, Troncoso MF, Gonzalez M, et al. Efficacy of different treatment alternatives for obesity hypoventilation syndrome. Pickwick Study. Am J Respir Crit Care Med. 2015;192(1):86-95

6. Budweiser S, Riedl SG, Jörres RA, Heinemann F, Pfeifer M. Mortality and prognostic factors in patients with obesity-hypoventilation syndrome undergoing noninvasive ventilation. J Intern Med. 2007:261(4):375-83.

7. Priou P, Hamel J-F, Person C, Meslier N, Racineux J-L, Urban T, et al. Longterm outcome of noninvasive positive pressure ventilation for obesity hypoventilation syndrome. Chest. 2010;138(1):84-90.

8. Pérez de Llano LA, Golpe R, Ortiz Piquer M, Veres Racamonde A, Vázquez Caruncho M, López MJ, et al. Clinical heterogeneity among patients with Obesity Hypoventilation Syndrome: therapeutic implications. Respiration. 2008;75(1):34-9.

9. Banerjee D, Yee BJ, Piper AJ, Zwillich CW, Grunstein RR. Obesity hypoventilation syndrome: hypoxemia during continuous positive airway pressure. Chest. 2007;131(6):1678-84.

10. Salord N, Mayos M, Miralda RM, Farré A, Carreras M, Sust R, et al. Continuous positive airway pressure in clinically stable patients with mild-to-moderate obesity hypoventilation syndrome and obstructive sleep apnoea. Respirology. 2013;18(7):1135-42.

11. Piper AJ, Wang D, Yee BJ, Barnes DJ, Grunstein RR. Randomised trial of CPAP vs bilevel support in the treatment of obesity hypoventilation syndrome without severe nocturnal desaturation. Thorax. 2008;63(5):395-401.

12. Castro-Añón O, Pérez de Llano LA, De la Fuente Sánchez S, Golpe R, Méndez Marote L, Castro-Castro J, et al. Obesity-Hypoventilation Syndrome: Increased Risk of Death over Sleep Apnea Syndrome. PLoS ONE. 2015;10(2):e0117808.

13. NPPV Titration Task Force of the American Academy of Sleep Medicine. Best clinical practices for the sleep center adjustment of noninvasive positive pressure ventilation (NPPV) in stable chronic alveolar hypoventilation syndromes. J Clin Sleep Med JCSM Off Publ Am Acad Sleep Med. 2010;6(5):491-509.
14. Janssens J-P, Borel J-C, Pépin J-L, SomnoNIV Group. Nocturnal monitoring of home non-invasive ventilation: the contribution of simple tools such as pulse oximetry, capnography, built-in ventilator software and autonomic markers of sleep fragmentation. Thorax. 2011;66(5):438-45.

15. Miller MR, Hankinson J, Brusasco V, Burgos F, Casaburi R, Coates A, et al. Standardisation of spirometry. Eur Respir J. 2005;26(2):319-38.

16. Berry RB, Budhiraja R, Gottlieb DJ, Gozal D, Iber C, Kapur VK, et al. Rules for scoring respiratory events in sleep: update of the 2007 AASM Manual for the scoring of sleep and associated events. Deliberations of the sleep apnea definitions task force of the American Academy of Sleep Medicine. J Clin Sleep Med JCSM Off Publ Am Acad Sleep Med. 2012:8(5):597-619.

17. Ayappa I, Berge Kl, Norman RG, Oppenheimer BW, Rapoport DM, Goldring RM. Hypercapnia and ventilatory periodicity in obstructive sleep apnea syndrome. Am J Respir Crit Care Med. 2002;166(8):1112-5.

18. Norman RG, Goldring RM, Clain JM, Oppenheimer BW, Charney AN, Rapoport DM, et al. Transition from acute to chronic hypercapnia in patients with periodic breathing: predictions from a computer model. J Appl Physiol. 2006;100(5):1733-41.

19. Lin C. Effect of nasal CPAP on ventilatory drive in normocapnic and hypercapnic patients with obstructive sleep apnoea syndrome. Eur Respir J. 1994;7(11):2005-10.

20. Han F, Chen E, Wei H, He Q, Ding D, Strohl KP. Treatment effects on carbon dioxide retention in patients with obstructive sleep apnea-hypopnea syndrome. Chest. 2001;119(6):1814-9.

21. Steier J, Jolley CJ, Seymour J, Roughton M, Polkey MI, Moxham J. Neural respiratory drive in obesity. Thorax. 2009:64(8):719-25.

22. Schönhofer B, Köhler D. Effect of non-invasive mechanical ventilation on sleep and nocturnal ventilation in patients with chronic respiratory failure. Thorax. 2000;55(4):308-13.

23. Ojeda Castillejo E, De Lucas Ramos P, López Martin S, Resano Barrios P, Rodríguez Rodríguez P, Morán Caicedo L, et al. Noninvasive mechanical ventilation in patients with obesity hypoventilation syndrome. Long-term outcome and prognostic factors. Arch Bronconeumol. 2015;51(2):61-8.

24. De Miguel Díez J, De Lucas Ramos P, Pérez Parra JJ, Buendía García MJ, Cubillo Marcos JM, González-Moro JMR. Analysis of withdrawal from noninvasive mechanical ventilation in patients with obesity-hypoventilation syndrome. Medium term results. Arch Bronconeumol. 2003;39(7):292-7.

25. Mokhlesi B, Tulaimat A, Faibussowitsch I, Wang Y, Evans A. Obesity hypoventilation syndrome: prevalence and predictors in patients with obstructive sleep apnea. Sleep Breath. 2007;11(2):117-24.

26. Georges M, Adler D, Contal O, Espa F, Perrig S, Pépin JL, et al. Reliability of apnea hypopnea index measured by a home Bi-level Pressure support ventilator versus a polysomnographic assessment. Respir Care. 2015;60(7):1051-6.

\section{Submit your next manuscript to BioMed Central} and we will help you at every step:

- We accept pre-submission inquiries

- Our selector tool helps you to find the most relevant journal

- We provide round the clock customer support

- Convenient online submission

- Thorough peer review

- Inclusion in PubMed and all major indexing services

- Maximum visibility for your research

Submit your manuscript at www.biomedcentral.com/submit
) Biomed Central 